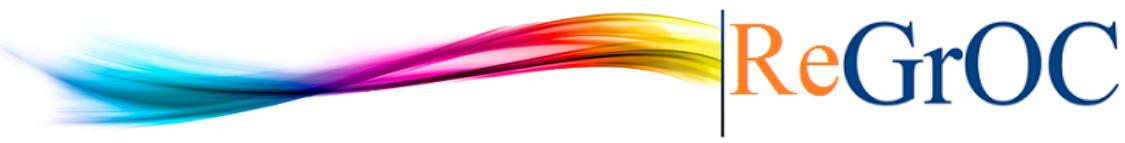

\title{
El análisis morfológico en el aula. Perspectiva, contenidos y actividades ${ }^{1}$
}

\author{
Irene Gil Laforga (Centro de Estudios de la Real Academia Española)
}

iregilo1@ucm.es

\section{RESUMEN}

En este trabajo se proponen diversas estrategias para acotar, reducir y graduar los contenidos de morfología que se abordan en la Enseñanza Media en España. En concreto, se sugiere adoptar una perspectiva única en el análisis morfológico que se lleva a cabo en Secundaria y Bachillerato, adaptar el grado de abstracción de la segmentación morfológica a cada nivel educativo y seleccionar para cada etapa las unidades léxicas que son objeto de estudio en el aula. Además, se proponen actividades en las que el análisis morfológico se combina con el semántico y el sintáctico, en una concepción de la morfología como un componente más del conocimiento de la lengua que el estudiante debe adquirir. Los ejercicios, que se adaptan a distintos niveles, están diseñados con el fin último de que los alumnos comprendan los mecanismos de creación e interpretación de la palabra compleja en su propia lengua.

\section{PALABRAS CLAVE}

Didáctica, morfología, actividades, perspectiva sincrónica, análisis morfológico, procesos de formación de palabras

\section{ABSTRACT}

This paper proposes several strategies to limit, reduce and graduate the morphological contents at Secondary Education in Spain. Particularly, I suggest to adopt a unique perspective for morphological analysis in order to adapt the degree of abstraction for morphological division to each educational level and to select a subgroup of lexical units appropriate for each period. Moreover, the paper proposes some activities where morphological analysis is combined with semantic and syntactic ones, since Morphology is conceived as one of the component of linguistic knowledge that every student must acquire. These exercises, that are adapted to each level, are designed so that students

\footnotetext{
${ }^{1}$ Quiero dar las gracias a los organizadores y a los asistentes de las I Jornadas GrOC (Gramática Orientada a las Competencias) Aragón por sus preguntas y comentarios sobre algunos de los aspectos que trata este artículo. Gracias también a Josefa Martín García por sus acertadas observaciones a una versión previa de este trabajo y a los revisores anónimos de esta revista por sus comentarios y sugerencias.
} 
are able to understand procedures of creation and interpretation of complex words in their own language.

\section{KEY WORDS}

Teaching, Morphology, activities, synchronic perspective/point of view, morphological analysis, word formation processes

\section{ÍNDICE}

\section{Introducción \\ 2. Sincronía y diacronía en el análisis morfológico \\ 3. La segmentación morfológica \\ 4. Algunos problemas del análisis morfológico \\ 5. Propuesta de actividades \\ 6. Conclusiones \\ 7. Referencias}

\section{Introducción}

Uno de los retos a los que se enfrenta el docente de lengua en el aula de Secundaria y Bachillerato es el modo de abordar las ingentes listas de objetivos que se formulan en los textos oficiales. Como han señalado Bosque y Gallego (2018), estas listas suponen una especie de "brindis al sol" pues establecen grandiosos objetivos de fácil formulación sin tener en cuenta «la enorme dificultad que existe para lograr que los estudiantes los alcancen o los profesores los transmitan en el trabajo diario en las aulas» (p. 155).

La morfología, dentro del ámbito de lengua, no constituye una excepción. Los siguientes contenidos forman parte del Bloque 3 de Conocimiento de lengua de primer ciclo de la $\mathrm{ESO}^{2}$ :

I. Elementos constitutivos de la palabra compleja

II. Procedimientos para formar palabras

Hasta donde conocemos, ninguna tesis doctoral en la actualidad es tan ambiciosa en sus objetivos. De hecho, ambos aspectos sirven para definir el objeto de estudio de una (sub)disciplina como la morfología léxica. Así, es adecuado afirmar que la morfología léxica se ocupa del estudio de los «elementos

\footnotetext{
${ }^{22}$ BOE Real Decreto 1105/2014, de 26 de diciembre.
} 
constitutivos de la palabra compleja» y de los «procedimientos para formar palabras».

Tal como se presentan los contenidos en este documento lo que se pretende alcanzar en esta etapa educativa es «reconocer, usar y explicar» todos los procedimientos de formación de palabras, sin establecer ningún límite. Como se ha señalado, este afán de exhaustividad conduce a la falsa creencia de que todo se puede analizar, cuando la propia teoría lingüística no ha conseguido dar respuesta a ciertos fenómenos o no ha llegado a acuerdos sobre sus análisis.

En este contexto, el presente trabajo pretende contribuir a afrontar el análisis morfológico en la Enseñanza Media a través de las siguientes propuestas:

i) Acotar: elegir una sola dimensión para el análisis de la palabra compleja.

ii) Graduar: no solo los contenidos se deben adaptar a los distintos niveles, sino también las exigencias. Se propone aumentar progresivamente el grado de abstracción del análisis en las distintas etapas educativas.

iii) Reducir: no todas las palabras se pueden analizar. Es necesario reconocer los problemas intrínsecos de la disciplina y ver cuáles se pueden abordar en el aula y en qué nivel.

Estas propuestas se integran en el artículo del siguiente modo: en la sección 2 se revisan las posibles aproximaciones al análisis morfológico y se señalan las diferencias del enfoque sincrónico y del diacrónico, así como las principales ventajas de cada perspectiva. La sección 3 se ocupa de la segmentación morfológica: se presenta como un proceso natural e intuitivo (§ 3.1) que se puede desglosar en tres pasos (§ 3.2) y con distinto grado de profundidad (§ 3.3). En la sección 4 se señalan algunos retos que plantea el estudio de la morfología y que es necesario tener en cuenta a la hora de elegir las palabras que se analizan en el aula. Finalmente, en la sección 5 se proponen algunas actividades orientadas a fomentar la observación, la reflexión y, en último término, la comprensión de la palabra compleja. 


\section{Sincronía y diacronía en el análisis morfológico}

En este apartado pretendemos ilustrar cómo el análisis de la estructura de las palabras puede abordarse desde dos puntos de vista, sincrónico y diacrónico, y cómo especialmente en el aula, se hace necesario el estudio diferenciado de ambas perspectivas ${ }^{3}$.

Aunque ambos enfoques coinciden en el objeto de estudio, incluso en el tipo de preguntas que se plantean, los objetivos que persiguen y la metodología de análisis son diferentes. Estas diferencias se hacen explícitas en las siguientes citas de la Nueva Gramática de la Lengua Española [NGLE, en adelante]:

口 Punto de vista diacrónico: «evolución que experimentaron las formas latinas, así como la progresiva incorporación a nuestra lengua de neologismos de muy variado origen, por causas diversas, a lo largo de toda su historia».

Punto de vista sincrónico: «pautas morfológicas que permiten construir las formas compuestas y derivadas a las que los hablantes tienen acceso». 4

[NGLE § 1.6c]

Las palabras en cursiva representan los elementos más significativos de cada enfoque. Lo interesante desde la perspectiva diacrónica es determinar el origen de una voz, su evolución a lo largo de la historia y explicar las causas de los cambios experimentados. En cambio, desde una perspectiva sincrónica, interesa la estructura de la palabra en un momento determinado (que suele coincidir con el del estudio), para determinar qué pautas morfológicas emplean los hablantes para construir y comprender las palabras complejas.

\subsection{Distintos resultados en el análisis morfológico}

La primera consecuencia del estudio de la palabra compleja desde un enfoque sincrónico y uno etimológico es que ofrecen resultados diferentes. Para comprobarlo, en este apartado abordaremos el análisis morfológico de los adjetivos doloroso, asqueroso y asombroso desde ambos puntos de vista.

\footnotetext{
3 La misma idea aplicada al análisis teórico de la morfología está en los trabajos de Martín y Varela (2012) y Serrano Dolader $(1995,2012)$.

4 La cursiva es nuestra.
} 
Si realizamos un análisis sincrónico, observamos en primer lugar que los tres adjetivos comparten un componente del significado que indica causa, que se puede atribuir al sufijo -oso. Así, la paráfrasis definitoria común a las tres formaciones sería «que causa/provoca/produce $\mathrm{N}$ », siendo $\mathrm{N}$ el nombre de la base. Estos valores se manifiestan, por ejemplo, en los siguientes sintagmas: un recuerdo doloroso ('que causa dolor'), un olor asqueroso ('que provoca asco') y una actuación asombrosa ('que produce asombro'). A partir de estas consideraciones semánticas se pueden proponer las siguientes estructuras:

(1) $\left[[\text { dolor }]_{\mathrm{N}-\mathrm{O} O \mathrm{O}}\right]_{\mathrm{A}}$;

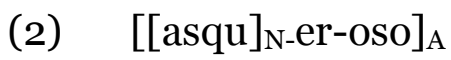

(3) $[\text { asombr }]_{\mathrm{N}-\mathrm{OSO}]_{\mathrm{A}}}$

Las segmentaciones de (2) y (3) no están exentas de polémica. En (2), el constituyente -er- que hemos aislado del sufijo -oso se considera un interfijo que se adjunta entre la base nominal asco y el sufijo -oso. Podría pensarse que se trata del mismo constituyente que figura, por ejemplo, en el derivado voltereta, que une la raíz sin diptongo volt- y el sufijo -eta. No obstante, también cabría considerar los análisis de (4) y (5):
(4) $\left[[\mathrm{asqu}]_{\mathrm{N}-\mathrm{eroso}}\right]_{\mathrm{A}}$

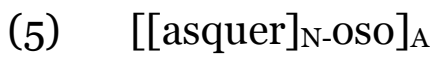

La segmentación de (4) asumiría que -eroso es un alomorfo del sufijo -oso. Dicho alomorfo se podría identificar en otros derivados como temeroso y valeroso. Otra posibilidad, representada en (5), es considerar que asquer- es un alomorfo de la raíz asc- ([ask-]). Esta segmentación no parece, en principio, la más adecuada, pues no resulta muy económico proponer la existencia de un alomorfo para un único derivado5.

También asombroso admite análisis alternativos al de (3), como el que se representa a continuación:

(6) $\left[[\text { asombr }]_{\mathrm{V}}-\mathrm{Oso}\right]_{\mathrm{A}}$

Como se observa en (6), esta segmentación es formalmente idéntica a la de (3) y la diferencia reside en la categorización de la base. El significado del adjetivo también se puede codificar recurriendo a una paráfrasis con el verbo asombrar: «que asombra». Más adelante veremos cuál de los dos análisis resulta más adecuado.

5 asquerosidad y asquerosamente derivan directamente de asqueroso, no de este supuesto alomorfo. 
Junto a estos análisis, pero desde un punto de vista diacrónico, existe otro modo de analizar estos derivados. El adjetivo doloroso procede del adjetivo latino dolorōsus con el que comparte significado. Desde esta perspectiva se trataría de un adjetivo simple en español, pues la adjunción del sufijo no se produce en esta lengua, sino en latín, a partir de la base dolor, doloris ${ }^{6}$. Esta evolución se representa en (7):

(7) doloroso < dolorōsus < dolor, ōris

Con este punto de vista, también es simple en español el adjetivo asqueroso que se encuentra en latín vulgar en la forma *escharosus 'lleno de costras', que, según se indica en $D C E C H$, se forma sobre el sustantivo eschara 'costra'. En este caso, el contenido semántico del derivado se explica a través de un cambio semántico 'lleno de costras' > 'que produce asco'. Esta derivación se representa en el esquema de (8):

(8) asqueroso < *escharosus < eschăra 'costra'

En una aproximación de este tipo, el adjetivo asombroso es complejo en español, pero tiene una complejidad mayor a la identificada en las segmentaciones de (3) y (6). El adjetivo procede del verbo asombrar y este deriva a su vez del sustantivo sombra. En el s. XIV, según el DCECH, el verbo asombrarse significa 'espantarse las caballerías por la aparición de una sombra'. Ya en el XV, el significado se generaliza, esto es, pierde parte de su intensión, y se emplea con el valor que tiene hoy de 'espantarse'. En (9) se ilustra esta evolución:

(9) asombroso < asombrar < sombra

En suma, el contraste entre las derivaciones de (1) a (3) y las de (7) a (9) ilustran que la estructura morfológica de una voz no coincide necesariamente con su etimología.

\subsection{Distintas respuestas a las mismas preguntas}

Además de las diferencias en la segmentación morfológica, los distintos enfoques ofrecen respuestas dispares a ciertas preguntas que se pueden formular explícita o implícitamente relacionadas con la estructura de las palabras. Dedicamos este apartado a ilustrar a través de ejemplos las distintas respuestas que pueden

\footnotetext{
${ }^{6}$ Alemany (1917: 566) «solo debiéramos considerar como voces derivadas en nuestra lengua, las que ella haya formado [...] y no las que ha recibido formadas ya del latín, como creador, de creatorem».
} 
ofrecer el análisis sincrónico y el diacrónico a las cuestiones que iremos formulando.

1) La complejidad de la palabra

Como hemos visto, una pregunta como «La palabra doloroso ¿̇es simple o compleja?» puede tener dos respuestas correctas. Desde el punto de vista sincrónico es compleja, se forma sobre el sustantivo dolor y tiene una estructura similar a ruidoso o angustioso. Sin embargo, en diacronía es un adjetivo simple en español pues se introduce en la lengua una vez formado.

Serrano Dolader (2012) ofrece ejemplos del mismo tipo para los sustantivos tristeza y traducción: tristeza es complejo si se relaciona sincrónicamente con triste o simple si se considera procedente de tristitia. A su vez traducción es complejo en sincronía, pues deriva de traducir, pero simple en diacronía, pues se incorpora como sustantivo procedente del latín traductio, ōnis.

2) La identificación del primitivo

De nuevo, cuando tratamos de identificar el primitivo del adjetivo asqueroso, también son posibles dos respuestas. Como hemos explicado, en sincronía este adjetivo se relaciona con una base nominal asco. Sin embargo, desde una perspectiva diacrónica, el adjetivo asqueroso es un primitivo, pues se introduce directamente procedente del latín.

3) La dirección derivativa

La cuestión sobre la dirección derivativa surge fundamentalmente en pares de verbo y nombre en los que no hay elementos formales (sufijos explícitos) que ayuden a la identificación de la base. Es el caso de la relación entre asombrar y asombro. Para ejemplos de este tipo, en sincronía se recurre al significado con el fin de determinar cuál es la base y cuál el derivado, de manera que se considera primitivo el término que no está incluido en la definición del otro. Según este criterio, el verbo asombrar no puede ser el primitivo, pues contiene en su definición el significado del sustantivo asombro: asombrar es causar asombro. En cambio, para definir asombro 'gran sorpresa o admiración' no es necesario recurrir al contenido del verbo. Por tanto, desde el punto de vista sincrónico, asombro es la base del verbo asombrar. 
Sin embargo, en un enfoque diacrónico se considera que la base corresponde a la categoría que se documenta primero en la lengua que, en este caso, según se indica en el $D C E C H$ es el verbo asombrarse ${ }^{7}$.

4) El número de morfemas

Las respuestas también son distintas a preguntas como la siguiente: «¿Cuántos morfemas tiene la palabra cafetera?». Un enfoque sincrónico distinguirá tres constituyentes: la base cafe-, el interfijo -t- y el sufijo -era. Se otorga en este caso una estructura análoga a la que identificamos en tetera. Sin embargo, en diacronía, la base de la que deriva cafetera no es café sino cafeto, de modo que solo habría dos morfemas: la base cafet (o) y el sufijo -era.

5) La naturaleza de los morfemas

De nuevo, si planteamos qué tipo de morfema es la terminación -ificar obtendremos respuestas diferentes. En sincronía se considera un sufijo derivativo verbalizador, mientas que en diacronía se puede considerar un elemento compositivo o, incluso, un verbo (facĕre). En el mismo sentido, Martín y Varela (2012) señalan la distinta consideración que reciben ciertos constituyentes iniciales como cosmo-, crono- o neuro-, que etimológicamente se denominan prefijos (Rainer 1993), mientras que en sincronía son elementos compositivos cultos.

\section{3. ¿Qué enfoque adoptamos en el aula?}

Hasta aquí hemos visto que hay dos enfoques posibles para analizar palabras y que ambos pueden ofrecer respuestas opuestas, de modo que es necesario diferenciar en el aula el análisis sincrónico y el diacrónico. La cuestión que puede surgir en este punto es qué perspectiva adoptamos cuando hacemos "análisis morfológico" en clase. La respuesta no es trivial y dependerá de cuál sea nuestro objetivo.

Son indudables las ventajas que tiene para el conocimiento de la lengua el estudio de su evolución. En primer lugar, como señalan Martín y Varela (2012), el cambio lingüístico permite una mejor comprensión del sistema lingüístico. Ciertos fenómenos que hoy se perciben como vulgares responden a procesos

\footnotetext{
7 Una búsqueda rápida en CORDE ofrece resultados en el mismo sentido: el verbo asombrar se documenta a finales del s.XIV-principios del XV, mientras que los ejemplos con el sustantivo no aparecen hasta un siglo después. El paréntesis etimológico del DLE apunta el mismo origen: asombrar_(de sombra), asombro_(de asombrar).
} 
sistemáticos que históricamente han dado lugar a la formación de palabras. Así, en la realización cocreta que se considera vulgar se produce el mismo proceso de metátesis que dio lugar a la actual forma murciélago. Según se ha señalado, esta voz procede del latín mūs, mūris y caecŭlus, diminutivo de caecus 'ciego'. La etimológica sería murciégalo, pero en la secuencia actual se han intercambiado las consonantes de las dos últimas sílabas.

Además, el estudio diacrónico tiene ventajas concretas en el ámbito de la morfología. Por ejemplo, ciertos derivados que se consideran irregulares en sincronía son perfectamente regulares en diacronía. Es el caso de los sustantivos detergente y suavizante. Un estudio sincrónico determinaría que la primera es opaca o se forma sobre la base opaca deterg-, mientras que la segunda es un adjetivo deverbal formado con la base verbal suaviza- y el sufijo -nte. En cambio, en diacronía se les asigna la misma estructura: detergente y suavizante proceden de antiguos participios de presente de los verbos deterger y suavizar.

A pesar del indudable interés que tiene el acercamiento diacrónico, si nuestro objetivo es mostrar el modo en que se procesan y generan las unidades léxicas, no parece que este sea el enfoque más adecuado. El motivo, según apuntan Martín y Varela (2012), es que «las razones históricas no tienen, en principio, incidencia en nuestro modo de procesar y generar las unidades léxicas». De hecho, «la competencia morfológica es a menudo contraria a la idea del peso histórico en los procesos morfológicos». Prueba de ello son los casos de etimología popular y ciertas peculiaridades del lenguaje infantil.

Se denomina etimología popular of falsa etimología a la deformación que realiza un hablante de una voz para asemejarla a otra con la que se relaciona semánticamente. Este proceso se identifica en las formas andalia (en lugar de "sandalia" por su relación con el verbo andar) y el conocido vagamundo (en lugar de vagabundo). En este último ejemplo, el cambio formal otorga una estructura morfológica muy frecuente en español, la de los compuestos VN.

Un ejemplo de competencia morfológica contraria a la forma etimológica se identifica en las producciones infantiles de formas regulares de participio en determinadas etapas del desarrollo del lenguaje. El conocimiento del paradigma flexivo lleva a producir los participios hacido, por hecho y ponido por puesto, entre otros. 
Por tanto, si el objetivo último del conocimiento de lengua es contribuir a descubrir el modo en que funciona el lenguaje, parece que el camino es la sincronía. En el siguiente apartado se trata la segmentación morfológica como método básico del análisis morfológico en sincronía.

\section{La segmentación morfológica}

Identificar las unidades mínimas de análisis es una práctica que resulta metodológicamente adecuada al abordar cualquier disciplina. Como hemos visto, la ley educativa actual establece que uno de los objetivos del bloque de Conocimiento de la lengua es el reconocimiento, uso y explicación de los elementos constitutivos de la palabra compleja. La identificación de dichos elementos se realiza a través de la segmentación morfológica.

Antes de explicar el modo en que se puede llevar a cabo este proceso, introduciremos dos consideraciones previas que prueban que la segmentación morfológica es natural e intuitiva. A continuación, veremos la manera de segmentar una palabra compleja. Más adelante propondremos modos de acotar un contenido tan abarcador.

\subsection{Un proceso natural e intuitivo}

La segmentación, entendida como la fragmentación de una secuencia lingüística, es un proceso natural que realizan los hablantes intuitivamente. La división del discurso en fragmentos menores que favorezcan su asimilación se lleva a cabo desde las primeras etapas de la adquisición del lenguaje.

O' Grady (2010 [2005]) recoge una prueba experimental que llevaron a cabo Saffran, Aslin y Newport (1996) con niños de 18 meses. En el experimento los niños oyen fragmentos de discurso con secuencias aleatorias durante dos minutos. Transcurrido este tiempo, oyen palabras inventadas formadas por tres sílabas. Algunas de estas palabras estaban formadas por secuencias ya oídas en los fragmentos del primer discurso. Estos autores observan que los niños tendían a girar la cabeza con mayor frecuencia al oír las palabras constituidas por 
secuencias oídas con antelación. El hecho de que un pequeño gire la cabeza constituye una señal de que ha percibido algo.

No solo la fragmentación es intuitiva. También lo es la segmentación morfológica, es decir, la división de palabras en morfemas. Tanto es así, que los hablantes tienden a identificar estas unidades incluso donde no las hay, como muestran ciertos casos de reanálisis. Los ejemplos que veremos a continuación ilustran cómo los hablantes reinterpretan la estructura de ciertas palabras identificando morfemas que no se encuentran en la estructura original de las voces.

En el primer caso, los hablantes identifican un constituyente derivativo, el prefijo bi-, en la voz bikini, que procede del topónimo Bikini. La prueba de este reanálisis son las voces creadas analógicamente, monokini y trikini, en las que el constituyente inicial designa el número de piezas del traje de baño.

En el segundo ejemplo, el hablante identifica unidades flexivas. En la red social Twitter, una periodista comenta el modo en que su abuela utiliza cierta expresión.

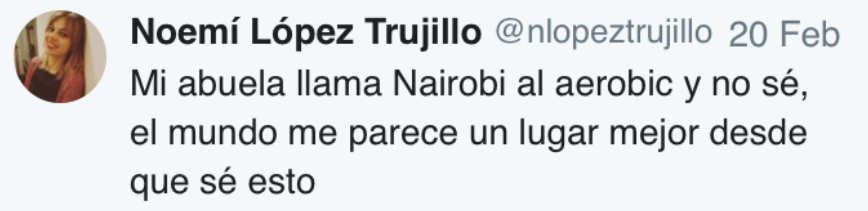

Figura 1

Este mensaje desencadena la respuesta de otros usuarios en los que comentan términos o expresiones de sus abuelos. Nos interesa especialmente una de estas respuestas:

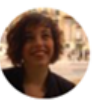

\section{Laura Casielles}

@lauracasielles

De mi abuelo, entre mis preferidos están los singulares lógicos: de sugus, "un sugo" ; de kleenex, "un clin"...

Figura 2

Según se desprende del tuit, el abuelo de su autora reinterpreta la terminación -es de [klínes] como el morfema de plural que se añade a los monosílabos terminados en consonante, de manera que el singular sería [klín]. También 
considera la terminación -us de sugus como un morfema de plural, por ello el singular es sugo ${ }^{8}$.

El tercer ejemplo también procede de Twitter. Esta vez, el autor del tuit crea un juego de palabras otorgando la estructura compositiva de un compuesto nominal formado por dos sustantivos al término buhardilla:

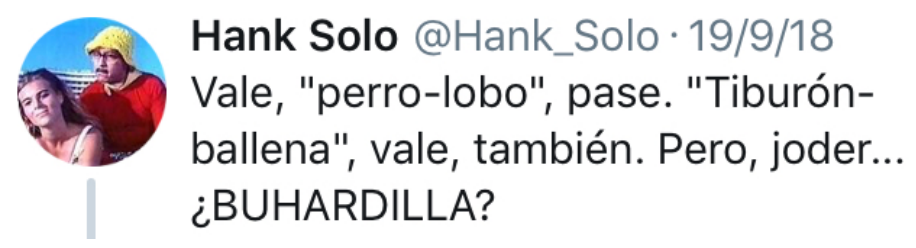

Figura 3

Estos casos muestran cómo tanto en el ámbito derivativo como en el flexivo, los hablantes tienden a identificar unidades morfológicas incluso donde, en principio, no las hay. En el siguiente apartado desgranaremos las fases en las que se lleva a cabo este procedimiento.

\subsection{La segmentación en tres pasos}

La introspección característica del análisis lingüístico nos conduce a establecer tres fases en el proceso de segmentación morfológica. La primera consiste en emparejar forma y significado. Identificar las partes constitutivas de una palabra pasa por asignar a cada fragmento un significado, sea este léxico, gramatical o relacional (v. arriba). Así, si segmentamos la voz negociador, inmediatamente identificamos un constituyente final -dor al que hacemos responsable de una parte del significado de la palabra 'persona que'.

Esta asociación se fundamenta en la "recurrencia" del sufijo, es decir, en su capacidad para producir voces en las que aporta el mismo significado. Así, la segunda fase de la segmentación morfológica consiste en buscar otras palabras en las que el constituyente identificado otorgue el mismo valor. En el caso propuesto, el sufijo aporta el mismo valor en otros términos como vendedor, reponedor, certificador, etc.

Por último, para confirmar que la división es adecuada, comprobamos que el elemento final es intercambiable por otros. En el caso de negociador, es posible sustituir el sufijo -dor por -ble o por -nte: negociable, negociante. Así, la tercera

\footnotetext{
${ }^{8}$ Según nos confirma la autora del tuit [c. p.], el hablante es de la zona occidental de Asturias. En esa área se conserva cierta alternancia en los sustantivos masculinos entre - o y - $u$ finales, según recoge Fernández Ordóñez (2007), a partir de Neira (1991).
} 
fase de la segmentación morfológica consiste en comprobar la intercambiabilidad del constituyente identificado.

La segmentación se completa repitiendo las tres fases hasta que el último constituyente sea indivisible, esto es, hasta que no sea posible asignar significado a unidades menores. Por tanto, para completar la segmentación de negociador, sería necesario identificar los constituyentes - $a$ - y negoci-. El primero representa la categoría verbal. El sufijo - ar ${ }^{9}$ - por su forma en infinitivo- se añade a bases nominales a las que aporta, entre otros valores, el significado 'hacer N', siendo $\mathrm{N}$ el nombre de la base. El mismo contenido se identifica en los verbos entrevistar 'hacer entrevistas', presupuestar o coleccionar, entre otros. Además, el sufijo es intercambiable, como se observa en negociete o negociado, que comparten la misma base. Por último, identificamos el constituyente negoci- que, en este caso, aporta el contenido semántico fundamental del término trato o gestión comercial', de modo que conforma la raíz de la palabra.

\section{3 ¿Cómo (re)presentamos la segmentación morfológica?}

A pesar de que la segmentación morfológica se lleve a cabo desde el principio de la Educación Secundaria Obligatoria, no parece adecuado exigir el mismo nivel de profundidad en todos los cursos.

Un modo de graduar es diferenciar el grado de abstracción con el que se presentan los resultados de la segmentación morfológica en los niveles más bajos $\left(1 .^{\circ}\right.$ y $2 .^{\circ}$ de la ESO) y aumentar la complejidad a medida que se superan los cursos. Aquí establecemos tres niveles, representados en (10), (11) y (12), respectivamente:

(10) a. negociador $<$ negociar $<$ negocio

b. [[[negoci]a]dor]

(11) $\left[\left[[\text { negoci }]_{\mathrm{N}} \mathrm{a}\right]_{\mathrm{V}} \mathrm{dor}_{\mathrm{N}}\right.$

(12) a. $\mathrm{V}+-d o r>\mathrm{N}$ : 'persona que $\mathrm{V}$ '

b. $\mathrm{N}+-a r>\mathrm{V}$ : 'hacer N'

En (10) la exigencia consiste en determinar los distintos constituyentes, bien presentando la derivación como un proceso, como en (10a), bien representando la jerarquía de la segmentación, como en (10b). En (11), se incorpora a la

9 Es polémica la naturaleza sufijal del constituyente - $a$ - pues coincide con la vocal temática (- $a$-), considerada por algunos autores como afijo flexivo. 
estructura jerárquica la categorización de cada constituyente. En (12) se expresan de forma escueta las reglas de formación de palabras incluyendo la categoría de la base, y la categoría y el significado del derivado. En este punto es necesario un mayor grado de abstracción, quizá más propio de los estudiantes de Bachillerato.

\section{Algunos problemas del análisis morfológico}

El análisis morfológico no siempre resulta tan sencillo como el del término negociador. Incluso el análisis presentado para esta voz tiene aspectos controvertidos. Así, no sería extraño que un alumno cuestionase la adecuación de la primera regla de (12) ante términos tan comunes como secador o impresora. En este caso, el profesor podría reducir ligeramente la intensión de la glosa para ampliar su extensión y dar cabida a los derivados con significado instrumental, de manera que la regla de (12) para el sufijo -dor podría tener el aspecto de (13):

(13) V+ -dor > N: 'aquel(lo) que V'

Esta modificación podría no satisfacer a los estudiantes si caen en la existencia de la voz comedor con el mismo constituyente -dor. En tal caso, el profesor puede cambiar de nuevo la paráfrasis definitoria del sufijo para dar cabida a las designaciones de lugar:

(14) V+-dor > N: 'persona, instrumento o lugar (en el) que V'

La agrupación de tantos significados como recoge la paráfrasis de (14) podría llevar a plantear si el sufijo -dor es un constituyente polisémico, o si se trata en realidad de distintos sufijos homónimos. En este caso, el docente tiene la oportunidad de aplicar el estudio de la polisemia y la homonimia a las unidades morfológicas.

La situación descrita no es en absoluto excepcional. Son muchos los casos en los que no existe correspondencia unívoca entre forma y significado en las unidades morfológicas. Se enumeran a continuación algunos de los desajustes más frecuentes ${ }^{10}$.

Entre los fenómenos que afectan al significado, se encuentran los constituyentes que carecen de contenido semántico. Es el caso de ciertos interfijos como -ecpresente en la formación de algunos diminutivos con diptongo en la base como

${ }^{10}$ En Fábregas (2015) se revisan estos fenómenos desde un punto de vista teórico. 
viejecito. Esta forma se emplea en ciertas zonas del español, mientras que en otras se prefiere la forma viejito. No es posible, por tanto, asignar un significado a este constituyente. También se puede producir la situación opuesta, es decir, que un constituyente contenga al mismo tiempo más de un significado. En estos casos se habla de sincretismo. Este fenómeno se produce de forma prototípica en las desinencias verbales. Así, la terminación - $b a$ - aúna los valores de tiempo pretérito, aspecto imperfecto y modo indicativo. También es posible que los constituyentes morfológicos sean polisémicos, es decir, que puedan adoptar distintos valores normalmente en combinación con diferentes bases (cabezazo 'golpe dado con la cabeza' vs. cochazo 'coche grande y bueno'), aunque no necesariamente (librazo 'golpe dado con un libro' o 'libro extenso y bueno’).

También se produce falta de correspondencia entre forma y significado cuando un mismo contenido puede ser representado por formas distintas. Esto sucede típicamente en los alomorfos de un morfema, esto es, en las distintas manifestaciones formales de un mismo morfema que aparecen en distintos contextos. Las propiedades que describen cada contexto pueden ser de tipo fonológico, morfológico o etimológico, principalmente. Así, la naturaleza fonológica del constituyente inicial de la base determina la forma que adopta el prefijo in-, que ante consonante líquida $(/ \mathrm{r} /$ o $/ 1 /)$ se materializa como $i$-. También existe un condicionamiento fonológico en la aparición o no de ciertos diptongos: la diferencia de la naturaleza del núcleo silábico de la primera sílaba de contar y cuento se debe a la ausencia del acento en la primera y su presencia en la segunda. Entre los contextos morfológicos que explican la presencia de alomorfos encontramos algunos de tipo flexivo, como la conjugación: el morfema del pretérito imperfecto de indicativo es - $b a$ - en la primera conjugación, pero - $a$ en la segunda y en la tercera. El tiempo verbal determina la forma de la base del verbo tener: ten- para las formas del tema de presente y tuv-para el tema de pasado. A partir de ciertos condicionamientos de tipo morfofonológico es posible predecir la aparición de cierto alomorfo: el sufijo -ble adopta la forma -bil- ante sufijos derivativos (amables vs. amabilidad). En otras ocasiones, el momento en el que se introduce una voz en la lengua puede determinar la forma de sus morfemas. Así, iglesia y eclesiástico contienen en la raíz dos variantes del mismo morfema. También los prefijos, sub-, so- y son- se consideran variantes formales de un morfema: subrayar, sonsacar, soterrar. 
Otro caso que ilustra la falta de univocidad en la expresión de un contenido morfológico lo representan los parasintéticos. En estas formaciones la derivación se produce a través de la adjunción simultánea de un prefijo y un sufijo: en-ternecer, a-silvestr-ar, en-suciar-ar, a-noch-ecer. Algunos autores consideran, sin embargo, que no hay dos morfemas derivativos sino uno discontinuo al que otorgan el nombre de circunfijo.

Más polémica es la cuestión de que existan morfemas sin contenido fonológico. Es esta la definición de morfema cero que se propone en un ámbito teóricodescriptivo para otorgar un análisis paralelo por ejemplo a la forma plural de los sustantivos. Junto a los alomorfos -s y -es existiría un morfema $\varnothing$ que se adjunta a los nombres polisílabos terminados en -s en singular, como lunes: el lunes vs. los lunes-Ø. Menos frecuente es la aplicación de este concepto a la morfología derivativa. Para otorgar entidad formal a los cambios categoriales sin expresión formal, en ocasiones se recurre a los morfemas cero.

También resulta polémica la segmentación morfológica en los casos en los que se producen cambios en la forma al adjuntar un morfema. Así, la adjunción del sufijo -ista a la base tenis conlleva la supresión de la secuencia coincidente en base y sufijo, con el resultado tenista en lugar de *tenisista. Este fenómeno se conoce con el nombre de haplología.

Por último, no resulta evidente la segmentación de aquellas voces cuyo significado está "lexicalizado" o desmotivado, es decir, aquellas en las que el contenido no se puede construir a partir del de los constituyentes. La lexicalización es un concepto gradual, de modo que no todos los hablantes tienen la misma sensibilidad a la hora de identificar el valor de un afijo. Así sucede en el caso de la terminación -ble de amable, en cuyo significado, para la mayoría de los hablantes, no existe rastro del valor que aporta normalmente el sufijo ('que puede ser V'), frente a lo que suceden en otros derivados "composicionales" como alcanzable. Menos clara suele resultar la presencia de un sufijo -ón en derivados como cinturón o de -illo en altillo o bolsillo.

\section{1. ¿Qué palabras segmentamos en el aula?}

Vistas las dificultades que acarrea la identificación de algunos morfemas, la propuesta de este apartado es tenerlas en cuenta a la hora de valorar si cierto término resulta adecuado para ser analizado en el aula. Para lograr este objetivo, 
nos fijamos en los criterios que han sido establecidos para determinar si una palabra es derivada. Algunos autores vinculan la derivación con la posibilidad de identificar un primitivo. Se asume, por tanto, que toda palabra derivada procede de una palabra existente en la lengua. Según este criterio, amable sería derivada, pues se relaciona con el primitivo amar, pero afable no lo sería, pues no es posible identificar una palabra en español de la que proceda. Un segundo criterio exige que, además de identificar un primitivo, exista una relación formal o semántica con él. De nuevo, amable se relaciona formalmente con su primitivo, luego se considera que deriva de él. También sería derivado oculista, pues se relaciona semánticamente con el primitivo ojo, aunque no formalmente. El tercer criterio es el más exigente, pues apela a la necesidad de que la relación entre primitivo y derivado sea semántica y formal al mismo tiempo. Los dos ejemplos anteriores, amable y oculista, no cumplen alguno de los dos requisitos, frente a deseable y trombonista, por ejemplo, que son transparentes desde el punto de vista semántico y formal.

La tabla representa de forma gráfica la relación de los ejemplos anteriores con su primitivo.

\begin{tabular}{|c|c|c|c|c|c|c|}
\hline & afable & amable & deseable & agonista & oculista & trombonista \\
\hline relación formal & & & & & & \\
\hline relación semántica & & & & & & \\
\hline
\end{tabular}

Tabla I. Relación formal y semántica entre derivado y primitivo

La aplicación de esta propuesta en el aula consiste en presentar palabras como deseable y trombonista en los primeros niveles (primer ciclo de la ESO) y reservar las del tipo amable y oculista para niveles superiores (segundo ciclo de la ESO y Bachillerato). Las de tipo afable y agonista se pueden comentar, pero no parecen, en principio, propicias para la segmentación en clase.

\section{Propuesta de actividades}

En este apartado se presentan algunas actividades para llevar a cabo en las sesiones de morfología. En cada ejercicio se especifican qué competencias se pretenden potenciar. También sus posibles variantes para adaptarlas a distintos niveles. 


\subsection{Morfología y semántica}

Actividad 1. Asocia las palabras con su significado y elabora una oración con cada una de ellas. [La X representa la base del derivado]

viajón, guapetón, bailón, preocupón, narizón

a. 'muy $X$ '

b. 'gran $X$ '

c. '(el) que tiene un/una gran $X$ '

d. '(el) que (se) X mucho'

Objetivos:

$>$ Descubrir que parte del significado de una palabra aparece codificado en los afijos.

$>\quad$ Revisar el concepto de polisemia y homonimia y extenderlo a las unidades morfológicas.

En esta actividad se incluye al menos un término que admite dos interpretaciones: viajón se puede definir como un gran viaje o como un adjetivo/sustantivo de persona 'que viaja mucho'.

El ejercicio permite trabajar la recategorización adjetivo > sustantivo de las voces que caracterizan ciertos aspectos de las personas, especialmente cuando son propiedades negativas (NGLE § 13.7). También es interesante establecer generalizaciones sobre el tipo de definición que admite un sustantivo y un adjetivo.

Este ejercicio se puede variar aumentando la lista inicial para ofrecer más derivados de cada tipo y al mismo tiempo no facilitar las paráfrasis definitorias, de manera que sea el propio alumno quien las deduzca. ${ }^{11}$

\subsection{Aplicación de la tipología Bosque (2015) al ámbito de la morfología} Como se indica en Bosque y Gallego, el objetivo último de los ejercicios de la tipología Bosque 2015 es comprender y analizar las «propiedades del sistema interiorizado con el que damos forma a nuestros pensamientos y con el que comunicamos nuestras intenciones» (Bosque y Gallego, 2016: 66).

1) Ejercicios de análisis inverso

\footnotetext{
${ }^{11}$ En Varela (2018 [2005]) se proponen otros ejercicios del mismo tipo.
} 
En los ejercicios de análisis inverso el estudiante construye una secuencia - una palabra en el ámbito de la morfología- «que se ajuste a las condiciones fijadas por el profesor». El valor didáctico está en que este tipo de ejercicio «permite desarrollar la capacidad de abstracción de los estudiantes». (Bosque y Gallego, 2016:76).

Actividad 2. Manipule el sustantivo masculino inventado sirto para obtener:

a. Un nombre masculino plural

b. Un verbo que signifique "meter en sirtos"

c. Un adjetivo que signifique "con forma de sirto"

d. Un nombre femenino que signifique "lugar donde se guardan los sirtos"12

Objetivos:

$>$ Favorecer la experimentación con un objeto lingüístico.

$>$ Desarrollar la capacidad de abstracción.

> Relacionar el contenido de un morfema con su expresión formal.

$>$ Descubrir que la categoría de una palabra compleja está determinada por los sufijos.

2) Ejercicios de pares mínimos

TIPO 1

El objetivo de este tipo de ejercicios es determinar las diferencias de significado y de estructura entre dos términos que se distinguen por un solo aspecto. Como indican Bosque y Gallego (2016: 78), «una parte esencial de los ejercicios de pares mínimos consiste en 'leer semánticamente’ el análisis sintáctico» (en este caso, el morfológico).

Actividad 3. Explique las diferencias de significado de los siguientes pares:

a. despido

b. $\quad$ despedida

El par de la actividad 3 contiene términos adecuados con significados distintos. En este caso se representan nominalizaciones de un verbo con distintos sufijos. A

${ }^{12}$ Se trabajan contenidos parecidos en el ámbito de la flexión el ejercicio propuesto por Escandell y Leonetti (2011: 68). 
pesar de que ambos términos se definen en el $D L E$ como 'acción y efecto de despedir o despedirse', los significados son diferentes, pues el primero equivale a ‘destitución' y el segundo a 'acompañamiento en el momento de irse'. El alumno podrá deducir que esta diferencia deriva de los distintos significados del verbo en cada nominalización. En a., el sustantivo se forma sobre el verbo despedir con el significado de 'prescindir de los servicios [de una persona]'. Mientras que en b. el sustantivo se forma sobre el verbo despedir con el valor de 'decir adiós'.

La complejidad del ejercicio puede aumentar si a cada uno de los derivados se añade un determinante posesivo $(\mathrm{mi})$. En el primer caso, el posesivo representa el paciente, de manera que no son posibles secuencias como ${ }^{*}$ mi despido de Carmen en las que la presencia del complemento desencadena la interpretación agentiva del posesivo. En cambio, el segundo caso (mi despedida) admitiría distintas interpretaciones: $m i$ puede representar a la persona que dice adiós ( $M i$ despedida de Carmen), con interpretación subjetiva, o la que es despedida (Recuerdo la fiesta que hicieron en mi despedida), con interpretación objetiva.

TIPO 2

Los pares de tipo 2 contienen secuencias con distinto grado de aceptación ${ }^{13} \mathrm{o}$ gramaticalidad. El objetivo de este tipo de ejercicio es explicar la anomalía de una secuencia y por qué esta no afecta al otro elemento del par.

Actividad 4. Explique la diferente aceptación de las siguientes secuencias:
a. ruptura sentimental
b. $\quad$ *rotura sentimental
c. $\quad$ rotura de tendones
d. $\quad$ */?ruptura de tendones

En la actividad 4, se representan nominalizaciones deverbales construidas con distintos alomorfos de la base en combinación con un complemento que determina su interpretación. En este ejercicio las diferencias de gramaticalidad se explican al identificar la distinta interpretación del derivado en función del alomorfo de su base. El par (a., b.) ilustra la mejor aceptación de la base culta cuando la acción designada no es física, mientras que el contraste entre c. y d. representa la adecuación de la base popular para designar la acción o el efecto sobre cuerpos sólidos. Esta generalización es válida para otros pares que se

13 En la tipología Bosque (2015), una de las dos secuencias es agramatical. Sin embargo, hemos comprobado que la gramaticalidad de los ejemplos varía en función de las áreas lingüísticas. 
forman con el mismo sufijo, como apertura y abertura. No se puede extender, en cambio, a otros ejemplos, como hechura y factura.

Como hemos visto, el ejercicio del tipo 1 se puede transformar en uno de tipo 2 si añadimos, además del posesivo, un complemento como de Carmen, que desencadena la agramaticalidad del ejemplo a. De esta manera, el alumno debe explicar las distintas propiedades sintácticas que posee cada derivado. Como indican Bosque y Gallego (2016: 78) «una de las principales ventajas didácticas de los ejercicios de pares mínimos es el hecho de que permiten contrastar de inmediato la secuencia que se analiza con otra similar para la que no es adecuado el análisis que tan satisfactorio nos podría parecer en un primer momento».

\section{Conclusiones}

El trabajo que hemos presentado aborda la enseñanza de la morfología en el aula de Secundaria y Bachillerato. El punto de partida es la fijación en los textos oficiales de unos contenidos tan amplios y abarcadores que resultan suficientes para definir una disciplina gramatical. Ante esta situación, el docente debe tomar ciertas decisiones, entre otras, qué enfoque adopta para el análisis morfológico el aula, cómo segmenta la palabra compleja en los distintos niveles y con qué palabras trabaja. A lo largo del trabajo se pretende dar respuesta a cada una de estas cuestiones partiendo de tres propuestas generales: la necesidad de acotar el enfoque a partir del cual se analiza morfológicamente una voz, la importancia de graduar el nivel de análisis que se exige en cada curso y el interés de reducir las palabras que se pueden analizar en el aula.

En este trabajo se defiende la idea de que el enfoque sincrónico es el más adecuado para conocer los procedimientos internos de creación e interpretación de las piezas léxicas, por tanto, es también el más adecuado para el análisis morfológico en la Enseñanza Media. También se señala la naturalidad del proceso de segmentación morfológica: el hablante lleva a cabo de manera intuitiva la identificación de las unidades morfológicas a través de la asociación de cada segmento con su significado. A pesar de ser intuitivo, es posible desgranar el proceso en distintas etapas (identificación, recurrencia e intercambiabilidad). Los resultados de la segmentación pueden alcanzar distintos grados de 
abstracción, de manera que pueden ser distintos en los distintos cursos. Se sostiene, igualmente, que los problemas que surgen en el análisis de la palabra compleja hacen que no todas las palabras puedan ser objeto de estudio en el aula. Se ofrece, por ello, una propuesta sobre el tipo de palabras más adecuadas para cada ciclo.

La parte final del artículo contiene una propuesta de actividades. En el primer bloque se ofrece un ejercicio que permite trabajar la morfología en combinación con otra disciplina lingüística como es la semántica. Sintaxis, morfología y semántica no son bloques estancos, sino que se pueden (y se deben) trabajar combinados en el aula de Secundaria y Bachillerato. En la misma línea, el segundo bloque es un intento de aplicar la tipología de ejercicios de Bosque (2015) al ámbito de la palabra compleja. En concreto se adoptan dos de los subtipos recogidos en Bosque y Gallego (2016): los ejercicios de análisis inverso y los ejercicios con pares mínimos. Estos ejercicios están diseñados para que el estudiante aprenda a través de la experimentación, para que consiga elaborar y valorar generalizaciones a partir de los datos y que, en último término, logre tomar conciencia de cómo funcionan los mecanismos morfológicos en su propia lengua. Además, en las actividades presentadas se introducen variables que permiten adaptarlas a los distintos niveles educativos.

\section{Referencias}

ALEMANY, José. (1917). De la derivación y composición de las palabras en la lengua castellana. Boletín de la Real Academia Española IV: 564-597.

Bosque, Ignacio. (2015). Ejercicios de análisis sintáctico. Sus clases y su aprovechamiento didáctico. Curso Aplicacions de la gramàtica a l'aula de Secundària i Batxillerat, organizado por CLT-ICE. Barcelona, 29 de junio-3 de julio de 2015.

Bosque, Ignacio y Ángel GALLEGo. (2016). La aplicación de la gramática en el aula. Recursos didácticos clásicos y modernos para la enseñanza de la gramática. Revista de Lingüística Teórica y Aplicada 54/2: 63-83.

Bosque, Ignacio y Ángel GALLEGo. (2018). La gramática en la Enseñanza Media. Competencias oficiales y competencias necesarias. ReGroC | Revista de gramática orientada a las competencias 1/1: 141-201. 
COROMINAS, J. y J. A. PASCUAL (1980-1991): Diccionario crítico etimológico castellano e hispánico (DCECH). Madrid: Gredos.

ESCANDELL, V. y M. LEONETTI (2011): "El estudio de la lengua: comunicación y gramática”. En U. Ruiz Bikandi (coord.): Lengua castellana y literatura. Complementos de formación disciplinar. Barcelona: Graó

FÁBREGAS, A. (2013): La morfología. El análisis de la palabra compleja. Madrid: Síntesis.

FERNÁNDEZ-ORDÓÑEZ, Inés (2007): Del Cantábrico a Toledo: "El "neutro de materia" hispánico en un contexto románico y tipológico (segunda parte), Revista de Historia de la Lengua Española 2: 29-81.

MARTín, Josefa y Soledad VARELA. (2012). La relevancia de la diacronía para la teoría morfológica. En M. Campos Souto [et al.] (eds.) «Assi como es de suso dicho». Estudios de morfología y léxico en homenaje a Jesús Pena, 323-336. San Millán de la Cogolla: Cilengua (Grupo USC FILGA).

NEIRA, Jesús (1991): Función y origen de la alternancia -u/-o en los bables centrales de Asturias. Boletín de la Real Academia Española LXXI: 433-454.

O’ GRADY, W. (2010 [2005]): Cómo aprenden los niños el lenguaje. Madrid: Akal.

RAINER, Franz (1993): Spanishe Wortbildungslehre. Tubinga: Max Niemeyer.

SERRANO-DOLADER, David. (1995). Las formaciones parasintéticas en español. Madrid: Arco/Libros.

SERRANO-DOLADER, David. (2012). Tensiones entre sincronía y diacronía en la descripción de la morfología léxica del español: el caso de la Nueva Gramática de la Lengua Española (NGLE). En M. Campos Souto [et al.] (eds.) «Assi como es de suso dicho». Estudios de morfología y léxico en homenaje a Jesús Pena, 485-498. San Millán de la Cogolla: Cilengua (Grupo USC FILGA).

Real Academia Española y Asociación de Academias de la Lengua Española (2009): Nueva gramática de la lengua española (NGLE). Madrid: Espasa.

Real Academia Española y Asociación de Academias de la Lengua Española (2014): Diccionario de la lengua española (DLE). Madrid: Espasa.

VARELA, Soledad. (2018 [2005]). Morfología léxica: la formación de palabras. Disponible en https://morforetem.files.wordpress.com/2018/06/formacic3b3n-depalabras.pdf >

Textos oficiales consultados

Boletín Oficial del Estado (BOE) 
Real Decreto 1105/2014, de 26 de diciembre, por el que se establece el currículo básico de la Educación Secundaria Obligatoria y del Bachillerato. Disponible en < https://www.boe.es/boe/dias/2015/01/03/pdfs/BOE-A-2015-37.pdfs 